\title{
Impact of Age on Ovarian Response and IVF Outcome during Controlled Ovarian Hyperstimulation in Women from Gaza Strip
}

\author{
MAGED M. YASSIN, ${ }^{1}$ ABDEL MONEM H. LUBBAD, ${ }^{2}$ MOHAMMED M. LAQQAN, ${ }^{3}$ EMAN S. ALZMAILY4
}

\begin{abstract}
Background: Although age is an important factor in female fertility, not much date were focused on the relationship between age and ovarian response and in vitro fertilization (IVF) outcome. However, the female reproductive capacity varies with age.

Objective: To assess the impact of age on ovarian response and IVF outcome during controlled ovarian hyperstimulation in women from Gaza Strip.

Methods: This prospective cohort study consisted of 75 women attending IVF at Al-Basma Fertility Center in Gaza City. The number of oocytes and embryos were recorded for each female and the occurrence of pregnancy was followed for three months. The obtained data were computer analyzed using SPSS statistical package version I 8.

Results: The mean age of the study population was $29.2 \pm 5.9$ years. The total number of oocytes was significantly decreased with increasing age ( $F=3.932$ and $P=0.024)$.In this context Pearson correlation test exhibited negative significant correlation between women age and the number of mature oocyte $(r=-0.276, P=0.017)$. There was an inverse relationship between age and ovarian response $(F=6.773$ and $P=0.001)$, showing good response (9-16 oocytes) at mean age of $26.7 \pm 5.0$ years. When related to women age, IVF outcome showed that the chance of getting pregnant increased with decreased age $(F=4.278$ and $p=0.018)$.
\end{abstract}

Conclusion: The ovarian response and the chance of getting pregnancy were diminishing with ageing, implying that maternal age should by consider during IVF program.

Keywords: Female age, Ovarian response, In vitro fertilization outcome, Gaza Strip.

\section{Introduction}

Controlled ovarian hyperstimulation $(\mathrm{COH})$ refers to a regime of fertility medication that used to stimulate the development of multiple follicles of the ovaries in one single cycle, resulting in superovulation. Controlled ovarian hyperstimulation for in vitro fertilization (IVF) cycles is usually monitored by serum estradiol levels and pelvic ultrasonography with two purposes: (1) to obtain an adequate number of mature oocytes, and (2) to prevent the risk of severe ovarian hyperstimulation syndrome. ${ }^{1,2}$ However, maternal age is of a particular concern prior to $\mathrm{COH}$ in the general practice of IVF treatment. ${ }^{3,4}$

Female age is the single most important determinant of spontaneous as well as treatment-related conception, with a gradual decline in fertility especially after the age of 35

1. Professor of Physiology at Faculty of Medicine, The Islamic University of Gaza, Gaza Strip, Palestine.

2. Associate Professor of Pathology at Faculty of Medicine, The Islamic University of Gaza, Gaza Strip, Palestine.

3. Lecture at Faculty of Medicine, The Islamic University of Gaza, Gaza Strip, Palestine.

4. Technologist in Al-Basma Fertility Center

Correspondence: Prof. Maged M. Yassin, Professor of Physiology at Faculty of Medicine, The Islamic University of Gaza, Gaza Strip, Palestine.E-mail: myassin@ iugaza.edu.ps years. ${ }^{5,6,7,8}$ A putative cause of such decline in fertility was attributed to diminished ovarian reserve which refers to the number and quality of oocytes. ${ }^{9}$ Older women may be more likely to be diagnosed with unexplained infertility and that this is due to the negative effect of age on ovarian reserve. ${ }^{10}$ Nonetheless, the relationship between age and declining reproductive capacity is highly variable..$^{11,12}$

Although extensive research has been carried out globally on IVF to achieve pregnancy, ${ }^{3,8,9}$ just recently two studies have been conducted in Gaza Strip and published in this regard entitled "Anti-mullerian hormone as a predictor of ovarian reserve and ovarian response in IVF women from Gaza strip"13 and "Serum estradiol level as a predictor of ovarian response and pregnancy outcome during controlled ovarian hyperstimulation in women from Gaza Strip". ${ }^{14}$ However, there was no previous studies related age to $\mathrm{COH}$ or even to the IVF outcome. Therefore, the present study is the first one to assess the impact of age on ovarian response and IVF outcome during $\mathrm{COH}$ in women from Gaza Strip.

\section{Methods}

This prospective cohort study consisted of 75 women undergoing $\mathrm{COH}$ program and aged between 20-40 years without history of other diseases. The subjects were recruited 
from Al-Basma fertility Center in Gaza City in the period September 2011 to November 2011. Each patient gave informed consent to participate in the study. The criteria for inclusion were as follows: (i) age 20-40 years, (ii) regular menstrual cycle, (iii) not on hormone therapy for three months and (iv) have not been subject to surgical operation in the reproductive system. This study was approved by the Ethical Committee of the institution. All participants were guaranteed confidentiality, and only the principal investigator has full access to the data.

\section{In Vitro Fertilization Protocol}

There were two protocols used during ovarian hyperstimulation, the first one is long protocol, which relies on pre-stimulation pituitary down regulation using $\mathrm{GnRH}$ agonists in daily intermittent or depot formulations. The second one is short protocols using GnRH antagonist during the late follicular phase of the stimulation cycle have been utilized. Adding recombinant $\mathrm{LH}$ to recombinant FSH protocols, when starting antagonists, as a strategy to increase oocyte yield and improve pregnancy rates. Human chorionic gonadotropin (hCG) was injected at a dose of 5000 or 10,000 IU. Oocyte retrieval for IVF was then typically scheduled for 30-34 hr thereafter. Then the fertilized oocyte was placed in G1 media for 3-4 days then in G2 media before rewind. The vaginal sonography was performed in the second day of the menstrual cycle to assess the extent of the responsiveness of the ovary. The number of oocytes and embryos were recorded for each female and the occurrence of pregnancy was followed for three months.

\section{Statistical analyses}

Simple distributions of the study variables and cross tabulation were applied. One-way ANOVA test was used for evaluating the relation between age and qualitative and quantitative variables. Pearson correlation test was applied. The results in all the above mentioned procedures were accepted as statistically significant when the p-value was less than $5 \%(\mathrm{p}<0.05)$.

\section{Results}

The study population included 75 females who had seeking in IVF at Al-Basma fertility Center in Gaza City, Gaza Strip. The mean age was $29.2 \pm 5.9$ years. Clinical data showed that the majority of the interviewed woman 46 (61.3\%) claimed that their husbands were the main cause of infertility. Around half of population $38(50.7 \%)$ had undergone repeated IVF. The result of IVF was positive in $27(36.0 \%)$ of the study population.

As indicated in table I, the age was classified into three categories: d'25, 26-35 and $>35$ years. The total number of oocytes was significantly decreased with increasing age registering means of $11.0 \pm 3.8,9.3 \pm 5.7$ and $5.8 \pm 5.6$, at age groups d" $25,26-35$ and $>35$ years, respectively $(\mathrm{F}=3.932$ and $\mathrm{P}=0.024)$. This inverse relationship was exhibited in mature oocytes and number of embryo, but the change in their mean number among the different age groups was not significant. Pearson correlation test exhibited negative significant correlation between women age and the number of mature oocytes $(\mathrm{r}=-0.276, \mathrm{P}=0.017)$ figure $(1)$.

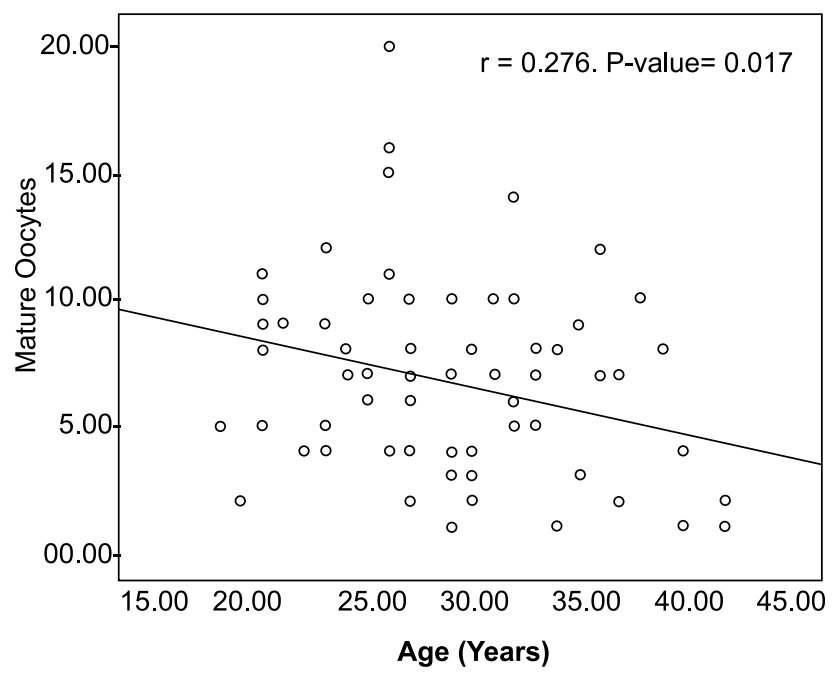

Fig.-1. Correlation between women age and the number of mature oocyte.

Table-I

Age classes of the study population in relation to various types of oocytes and embryos $(n=75)$

\begin{tabular}{lccccc}
\hline Oocytes & \multicolumn{3}{c}{ Age (year) } & F & P-value \\
\cline { 2 - 4 } & $(<25)(\mathrm{n}=20)$ & $(26-35)(\mathrm{n}=42)$ & $(>35)(\mathrm{n}=13)$ & & \\
\hline Total number of oocytes & $11.0 \pm 3.8$ & $9.3 \pm 5.7$ & $5.8 \pm 5.6$ & 3.932 & 0.024 \\
Mature oocytes & $7.4 \pm 2.7$ & $7.0 \pm 4.2$ & $4.5 \pm 3.8$ & 2.488 & 0.090 \\
Immature oocytes & $4.1 \pm 2.7$ & $2.7 \pm 2.6$ & $1.5 \pm 2.3$ & 3.971 & 0.023 \\
Number of embryo & $3.9 \pm 1.3$ & $3.6 \pm 1.6$ & $2.9 \pm 1.6$ & 1.781 & 0.176 \\
\hline
\end{tabular}

All values are expressed as mean $\pm \mathrm{SD}$.

P- value less than 0.05 was considered for statistical significance

$\mathrm{P}$ - value more than 0.05 was not considered for statistical significance 
Table II

Age of the study population in relation to ovarian response during IVF program $(n=75)$

\begin{tabular}{lccccc}
\hline Ovarian response & No. & $(\%)$ & Age (year) (mean \pm SD) & F & P-value \\
\hline Poor responders( $<4$ oocytes) & 15 & 20 & $33.4 \pm 6.5$ & 6.773 & 0.001 \\
Normal responders(4-8 oocytes) & 21 & 28 & $30.2 \pm 4.5$ & & \\
Good responders(9-16 oocytes) & 32 & 42.7 & $26.7 \pm 5.0$ & & \\
High responders( $>16$ oocytes) & 7 & 9.3 & $27.7 \pm 4.9$ & & \\
\hline
\end{tabular}

All values are expressed as mean $\pm \mathrm{SD}$.

P- value less than 0.05 was considered for statistical significance.

F: ANOVA test.

According to the number of oocytes retrieved upon stimulation by menotrophin (FSH 75IU, LH 75 IU), the study population was divided into poor, normal, good and high responders (Table II). Fifteen (20\%), 21 (28\%), 32 (42.7\%) and $7(9.3 \%)$ women gave $<4,4-8,9-16$ and $>16$ oocytes during ovarian stimulation of IVF program, respectively. When related to women age, the total number of oocytes was generally increased with decreased age, showing $<4,4$ $8,9-16$ and $>16$ oocytes at mean ages of $33.4 \pm 6.5,30.2 \pm 4.5$, $26.7 \pm 5.0$ and $27.7 \pm 4.9$ years, respectively. This inverse relationship between the total number of oocytes and age was found to be significant $(\mathrm{F}=6.773$ and $\mathrm{P}=0.001)$, implying that younger women yield more oocytes.

Table III shows the relationship between women age and IVF outcome. Positive pregnancy occurred in women aged $26.7 \pm 4.1$ years whereas negative pregnancy and no cleavage occurred at ages $30.6 \pm 6.4$ and $32.0 \pm 4.2$ years, respectively. When related to women age, IVF outcome showed that the chance of getting pregnant increased with decreased age $(\mathrm{F}=4.278$ and $\mathrm{p}=0.018)$.

Table III

Age of the study population in relation to IVF outcome during IVF program $(n=75)$

\begin{tabular}{llll}
\hline IVF outcome & $\begin{array}{l}\text { Age (year) } \\
(\text { mean } \pm \text { SD) }\end{array}$ & F & P-value \\
\hline Positive** & $26.7 \pm 4.1$ & 4.278 & 0.018 \\
Negative*** & $30.6 \pm 6.4$ & & \\
No cleavage & $32.0 \pm 4.2$ & & \\
\hline
\end{tabular}

All values are expressed as mean $\pm \mathrm{SD}$.

P- value less than 0.05 was considered for statistical significance. F: ANOVA test.

Positive: pregnancy occurred, Negative: no pregnancy.

\section{Discussion}

The results of this study indicated that the majority of women in the Gaza Strip seeking IVF when their husbands had fertility problems and the process of pregnancy is delayed. A total 75 women was enrolled in IVF programs. The mean age of women (29.2 years) was close to that reported in Egyptian (29.0 years), Iranian (29.1 years) and Palestinian (28.7 years) studies, ${ }^{13,15,16}$ but lower than that reported from the USA and South Korea (34.0 years). ${ }^{17,18}$ The younger women seeking IVF in developing countries, including Gaza Strip, could be explained in the context of social habits where most families have the desire to have children immediately after marriage and they encourage women to undergo IVF. This is supported by the finding that around half of the study population had undergone repeated IVF. The outcome of IVF was positive in $36.0 \%$ of the study population. This finding was in agreement with the results of studies from the Netherlands and South Korea which showed that $36.0 \%$ and $32.5 \%$ of women undergoing IVF achieved viable pregnancy. ${ }^{17,19}$ However, the pregnancy rate among patients who had an IVF trial in different settings was $23.1 \%$. Nevertheless, IVF treatment outcome is highly variable and difficult to predict. ${ }^{15}$

Data of the present study revealed an inverse relationship between women age and various types of oocytes, and embryo, with significant change detected with the total number of retrieved oocytes. Similar results were obtained. ${ }^{3,13,17,20}$ Such decrease in the total number of oocytes with aging may be related to increase basal level of FSH which results in diminishing ovarian reserve and to the responsiveness of women in IVF program. ${ }^{21,22}$ In this context, Pearson correlation test showed negative significant correlation between age and the number of mature oocyte. This result is in concurrent with that previously reported. ${ }^{13,20}$

Ovarian response was diminished with aging, showing poor response ( $<4$ oocytes) at the mean age of $33.4 \pm 6.5$ 
years and good response (9-16 oocytes) at the mean age of $26.7 \pm 5.02$ years. This means that younger women have a better chance of a successful IVF. Studies carried out on ovarian reserve and ovarian response in $I V F$ women found that the poor responses were detected at mean ages 36.3 and 32.5 years $^{23,24}$. This inverse relationship between ovarian response and female age was also documented in other studies. ${ }^{13,16,20}$ The IVF outcome showed that the youngest women (mean age $26.7 \pm 4.1$ years) had a better opportunity to get pregnant. This is supported by the aforementioned finding that ovarian response increased with decreasing age making more chance for pregnancy to occur. In addition, this finding coincides with other studies who found that pregnancy outcome of IVF program was significantly higher in younger women than that in the older ones. ${ }^{13,25}$ Similar findings were also reported, ${ }^{19}$ but the difference in the pregnancy outcome of IVF program was not significant.

\section{Conclusion}

In conclusion, the total number of retrieved oocytes, ovarian response and the chance of getting pregnancy were diminishing with ageing. Therefore, it is important to consider maternal age during IVF program.

\section{Conflict of Interest: None}

\section{References}

1. Theocharis P, Juliette G, Franc G, Catherine P, Yvonne F, Yvette J and Jean-Rene Z. Percentile curves of serum estradiol levels during controlled ovarian stimulation in 905 cycles stimulated with recombinant FSH show that high estradiol is not detrimental to IVF outcome. Human Reproduction 2002; 17(11): 2846-2850.

2. Gruber I, Just A, Birner M, and Losch A. Serum estradiol/ progesterone ratio on day of embryo transfer may predict reproductive outcome following controlled ovarian hyperstimulation and in vitro fertilization. Experimental \& Clinical Assisted Reproduction 2007; 4:1.

3. Tsung-Hsien L, Chung-Hsien L, Chun-Chia H, Kung- Chen $\mathrm{H}$, Pi-Mei L and Maw-Sheng L. Impact of female age and male infertility on ovarian reserve markers to predict outcome of assisted reproduction technology cycles. Reproductive Biology and Endocrinology 2009; 7:100.

4. Stern K. Assisted reproductive Technology. What's new and what's important?. Aust Fam Physician 2012; 41(10): 762-8.

5. Templeton A, Morris J, Parslow W. Factors that affect outcome of in-vitro fertilisation treatment. Lancet 1996; 348:1402-1406.
6. Chuang C, Chen C, Chao K, Chen S, Ho H, Yang Y. Age is a better predictor of pregnancy potential than basal follicle stimulating hormone levels in women undergoing in vitro fertilization. Fertil Steril 2003; 79:63-68.

7. Maheshwari A, Hamilton M, Bhattacharya S. Effect of female age on the diagnostic categories of infertility. Hum Reprod 2008; 23:538-542.

8. American Society for Reproductive Medicine. Age and Fertility, A Guide for Patients Revised 2012; 1-16.

9. Artini P, Ruggiero M, Uccelli A, Obino M and Cela V. Fertility Management of Patients with Reduced Ovarian Reserve. Reprod Sys Sexual Disorders 2013; 5:6

10. Gleicher N, Barad D. Unexplained infertility: Does it really exist?. Hum Reprod 2006;21:1951-1955.

11. te velde $\mathrm{R}$, and Pearson $\mathrm{L}$. The variability of female reproductive ageing. Human Reproduction 2002; 8: 141154.

12. Alviggi C, Humaidan P, Howles C, Tredway Donald and Hillier Stephen. Biological versus chronological ovarian age: implications for assisted reproductive technology. Reproductive Biology and Endocrinology 2009; 7:101.

13. Yassin M, Sharif F, Laqqan M. Anti-mullerian hormone as a predictor of ovarian reserve and ovarian response in IVF women from Gaza strip. Iran J Reprod Med 2013;11(4): 261-266.

14. Yassin M, Laqqan M, Alzmaily E. Serum Estradiol Level as a Predictor of Ovarian Response and Pregnancy Outcome During Controlled Ovarian Hyperstimulation in Women from Gaza Strip. J MEDICINE 2013; 14 : 52-56.

15. Shahin A. The problem of IVF cost in developing countries: has natural cycle IVF a place? Reproductive Bio-Medicine Online 2007; 15: 51-56.

16. Dehghani-Firouzabadi R, Tayebi N, Asgharnia M. Serum Level of Anti-Mullerian Hormone in Early Follicular Phase as a Predictor of Ovarian Reserve and Pregnancy Outcome in Assisted Reproductive Technology Cycles. Archives of Iranian Medicine 2008; 11:4.

17. Joo B, Park S, An B, Kim K, Moon S, and Moon H. Serum estradiol levels during controlled ovarian hyperstimulation influence the pregnancy outcome of in vitro fertilization in a concentration-dependent manner. Fertility and Sterility 2010; 93(2): 442-6.

18. Phelps J, Levine A, Hickman T, Zacur H, Wallach E, Hinton E. Day 4 estradiol levels predict pregnancy success women undergoing controlled ovarian hyperstimulation for IVF. Fertility and Sterility 1998; 69: 6. 
19. Smeenk J, Sweep F, Zielhuis G, Kremer J, Thomas C. and Braat D. Antimullerian hormone predicts ovarian responsiveness, but not embryo quality or pregnancy, after in vitro fertilization or intracyoplasmic sperm injection. Fertility and sterility 2007; 87(1): 223-226.

20. Ficicioglu C, Kutlu T, Baglam E, Bakacak Z. Early follicular antimüllerian hormone as an indicator of ovarian reserve. Fertility and Sterility 2006; 85(3): 592-596.

21. Karimzadeh M, Ghandi S. Age and basal FSH as a predictor of ART outcome. Iranian Journal of Reproductive Medicine 2009; 7(1):19-22.

22. Chaudhuri A, Chatterjee S. Selecting age-specific protocol for controlled ovarian hyperstimulation in IVF program with particular reference to advanced age group. Al Ameen J Med Sc i $2013 ; 6(1): 51-57$.
23. Van Rooij A, Broekmans J, Te Velde R, Fauser C, Bancsi F, De Jong H. and Themmen P. Serum anti-Mullerian hormone levels: a novel measure of ovarian reserve. Human Reproduction 2002; 17:3065-3071.

24. La Marca A, S.Giulini, Tirelli A, Bertucci E, Marsella T, Xella S. and Volpe A. Anti-Mullerian hormone measurement on any day of the menstrual cycle strongly predicts ovarian response in assisted reproductive technology. Human Reproduction 2007; 22(3):766-771.

25. Gnoth C, Schuring A, Friol K, Tigges J, Mallmann P, Godehardt E. Relevance of anti-Mullerian hormone measurement in a routine IVF program. Human Reproduction 2008; 23:1359-1365. 